\title{
Les plaisirs enchantés : célébration, fêtes jeux et joutes poétiques dans les jardins japonais de l'époque de Heian
} Michel Vieillard-Baron

\section{Résumé}

Dans le Japon de l'époque Heïan, le jardin est un espace théâtralisé où se déroulent cérémonies, banquets et jeux mettant en scène le pouvoir impérial. Il est aussi le lieu privilégié de l'expression de diverses formes artistiques : musique, danse, poésie. Il est, en particulier, le cadre de joutes poétiques d'origine chinoise, organisées à la faveur de réunions à boire au cours desquelles le saké était servi dans des tasses qu'on laissait flotter au fil de l'eau d'un petit ruisselet sinueux aménagé tout exprès dans le jardin.

\begin{abstract}
Delightful enjoyments in Heian period gardens

In Japan during the Heian period, the garden was a theatrical space where ceremonies, banquets, and games took place that displayed imperial power. It was also the place of choice for a variety of artistic expressions : music, dance, poetry. In particular, it provided the setting for the poetic tournaments of Chinese origin organized on the occasion of drinking parties where the sake was served in cups set to float with the current of small winding brooks specially laid out for the purpose.
\end{abstract}

\section{Citer ce document / Cite this document :}

Vieillard-Baron Michel. Les plaisirs enchantés : célébration, fêtes jeux et joutes poétiques dans les jardins japonais de l'époque de Heian. In: Extrême-Orient, Extrême-Occident, 2000, n²2. L'art des jardins dans les pays sinisés. Chine, Japon, Corée, Vietnam. pp. 93-114.

doi : $10.3406 /$ oroc. 2000.1118

http://www.persee.fr/doc/oroc_0754-5010_2000_num_22_22_1118

Document généré le 16/10/2015

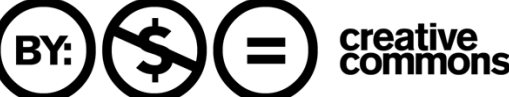




\title{
Les plaisirs enchantés : célébrations, fêtes, jeux et joutes poétiques dans les jardins à l'époque de Heian
}

\author{
Michel Vieillard-Baron
}

« [...] On descendra droit au parterre de gazon, on s'arrestera au bas de l'allée obscure pour considérer les jets qui l'environnent. On ira passer à la fontaine qui est dans le petit bosquet pour aller à la cascade basse. On remontera l'allée jusques à la haute. [...] On en descendra pour entrer dans les bateaux pour aller à l'Appolon [...] » écrivait entre 1702 et 1704 Louis XIV dans sa Manière de montrer les jardins de Versailles '. Dans ce texte, le roi nous sert de guide et nous indique, sur le ton autoritaire d'une ordonnance royale, l'itinéraire que l'on doit emprunter afin de goûter de la meilleure façon les beautés et les plaisirs du jardin. Si Louis XIV prit le soin d'écrire ce texte, - il ne le fit pas pour l'architecture du château -, c'est que les jardins, plus que tout autre élément du domaine de Versailles, constituaient, selon les termes de Jean-Pierre Babelon, « un champ d'action à son génie autrement développé, autrement satisfaisant, autrement cosmique ${ }^{2} \gg$. L'importance, tant esthétique que sociale, des jardins dans la culture occidentale - et française en particulier - n'est plus à rappeler ${ }^{3}$. Depuis la Renaissance, les jardins ont été le théâtre de nombreuses réjouissances dont l'histoire a parfois conservé le souvenir ${ }^{4}$. Dans un lieu magique et, tout comme les fêtes, éphémère, musique, danses, spectacles et feux d'artifices permettaient d'oublier, l'espace d'un instant, les contingences du monde.

Pour bien de nos compatriotes, il en va autrement au Japon. L'image la plus couramment diffusée étant celle des jardins de temples, les jardins japonais évoquent bien souvent dans l'esprit des Occidentaux des surfaces de sable soigneusement ratissées, parfois agrémentées de quelques ensembles de pierres brutes ou de végétaux rachitiques.... Il s'agit là d'une vision pour le moins réductrice : tous les jardins du Japon ne sont pas des jardins zen. Dans la présente étude, nous allons nous attacher à évoquer les différentes activités qui se déroulaient dans les jardins du palais impérial et des résidences de la noblesse à l'époque de Heian (784-1185) pour découvrir qu'avant d'être objets de contemplation, les jardins étaient des espaces de vie.

Les jardins de l'époque de Heian ${ }^{5}$ étaient conçus pour agrémenter les résidences de la noblesse ${ }^{6}$. Celles-ci se caractérisaient par une certaine symétrie dans le plan qui s'ordonnait autour du pavillon principal, shinden. La surface minimum pour une 


\section{Michel Vieillard-Baron}

résidence de ce type était un peu plus d'un hectare ${ }^{7}$. La propriété était entourée d'un mur de pisé - remplacé parfois par une enceinte de terre sur laquelle on plantait une haie vive - percé de portes sur trois faces, nord, est et ouest. Le pavillon principal s'étalait d'est en ouest, face au sud. Il était flanqué de deux pavillons, appelés respectivement pavillon de l'est et pavillon de l'ouest (un de ces pavillons pouvait être absent et remplacé par une galerie et une structure plus légère). Les pavillons étaient reliés par des galeries de communication. Il existait aussi, de pavillon à pavillon, des couloirs qui permettaient de circuler à l'abri et, au besoin, de placer des subalternes lors des cérémonies. Les couloirs qui, des pavillons de l'est et de l'ouest, se dirigeaient vers le sud, étaient percés en leur milieu d'une porte. Ces couloirs pouvaient conduire à divers bâtiments de service ou de plaisance, comme par exemple le pavillon de pêche ${ }^{8}$, tsuri dono, qui servait de lieu de réunions poétiques, de refuge contre les chaleurs estivales et d'embarcadère lors des promenades en bateau sur l'étang du jardin. Le pavillon principal faisait face à un espace libre - appelé " cour »-, assez vaste pour permettre les alignements et les saluts, prolongé par le jardin. Chaque pavillon - en fait un vaste espace couvert d'un toit soutenu par des poteaux - était surélevé et on y accédait par des degrés placés sur les diverses faces ; autour, une allée était à l'abri sous l'avancée du toit. Ouvert sur l'extérieur, le pavillon d'une résidence se trouvait en rapport étroit avec le monde végétal et minéral environnant. On pouvait par ailleurs trouver autour des bâtiments de petits jardins clos qui donnaient leur nom aux pavillons qu'ils longeaient : "clos aux glycines ", fuji tsubo, "clos aux pruniers », ume tsubo, etc. Le jardin était généralement établi autour d'un étang comportant une ou plusieurs îles, plus ou moins élevées, reliées par des ponts : pont en dos d'âne reliant l'île principale à la cour (son arche devait être suffisamment haute pour permettre le passage des barques et placée de biais afin que sa courbe pût être admirée depuis le pavillon principal), ponts plats ailleurs. L'étang était alimenté par un cours d'eau qui devait, en principe, prendre sa source au nord-est. Le cours d'eau pouvait être aménagé de manière à évoquer, par exemple, un torrent de montagne. Si le relief le permettait, on bâtissait une cascade. Dans le jardin, on pouvait reproduire - ou plutôt transposer en retenant uniquement les traits fondamentaux - certains lieux célèbres du Japon. Ainsi, dans le jardin du Kawara no in, la résidence de Minamoto no Tôru (822-895), l'étang fut conçu à l'imitation du fameux paysage de Shiogama, en Michinoku, dont la côte est parsemée d'îlots couverts de pins aux formes tourmentées ${ }^{9}$.

L'eau jouait un rôle essentiel dans l'agencement du jardin ${ }^{10} \mathrm{car}$ un réseau de cours d'eau aménagé selon les règles " devait assurer le confort des habitants de la résidence. La disposition de chacun des autres éléments du jardin : pierres, arbres, étang, îles, faisait également l'objet de soins méticuleux : il en allait, pensait-on, du bien-être des résidents.

Dans un espace aussi précisément ordonné, jardin et cour, étroitement associés aux bâtiments, servaient pour les célébrations et les divertissements. L'auteur du Sakutei-ki n'écrivait-il pas dans son traité : 
L'aménagement de la cour sud doit être fait de sorte que des piliers extérieurs de l'escalier couvert du pavillon principal au rivage de l'étang, il y ait six ou sept jô (soit dix-huit ou vingt et un mètres). S'il s'agit [de la cour sud] du palais impérial où se déroulent les cérémonies, l'espace doit atteindre huit ou neuf jô (soit vingt-quatre ou vingt-sept mètres), car il faut prévoir les salutations. [...]

Par ailleurs, pour ce qui est de l'édification de l'île, ce sera fait en fonction de la configuration de l'endroit, de l'étendue ou de l'étroitesse de l'étang. [...] Bien que je souhaite, étant donné que les tribunes des musiciens atteignent sept voire huit jô (soit vingt et un ou vingt-quatre mètres), que vous conceviez l'île large, comme cela dépend de l'étang, il faut installer une île légèrement en retrait par rapport à l'autre, de manière à pouvoir y poser les rallonges des estrades provisoires ${ }^{12}$.

Célébrations, jeux et fêtes, indissociables de la vie de la noblesse de ce temps, étaient, comme le révèle la citation du Sakutei-ki que nous venons de voir, inscrits dans la conception même du jardin. Examinons à présent en quelle occasion et de quelle façon l'aristocratie de Heian utilisait cet espace.

\section{Le jardin comme lieu de célébrations}

À l'époque de Heian, le « Cycle annuel des célébrations », nenjû gyôji, rythmait la vie de la cour et, plus largement, de la noblesse ${ }^{13}$. Ce cycle de célébrations - une centaine selon le décompte fait par Francine Hérail ${ }^{14}$-, comprenait procédures administratives, rites de cour et célébrations religieuses. Ces célébrations avaient une portée politique car elles étaient considérées comme nécessaires au bon gouvernement du pays. Elles se répartissaient de manière inégale sur les douze mois lunaires que comptait l'année : le premier et le dernier mois étaient les plus chargés. Certaines d'entre elles se déroulaient, au moins en partie, dans le jardin ou les cours, qui étaient assimilées à des jardins ${ }^{15}$. Dans sa minutieuse étude consacrée au Sakutei-ki ${ }^{16}$, Tamura Tsuyoshi recense quelque trente-deux célébrations qui, à son avis, avaient un quelconque rapport avec le jardin ${ }^{1}$. Il semble toutefois que ce chiffre soit excessif. Nous n'évoquerons donc ci-dessous que les célébrations qui, de manière attestée, se déroulaient à l'extérieur, dans le jardin d'un palais ou d'une demeure.

Le «Salut aux quatre points cardinaux », Shihôhai, est le premier rite accompli par l'empereur au matin du premier jour de l'année. L'empereur commençait par prendre un bain afin de se purifier, puis descendait sur le sol du jardin. Là, tourné vers le nord, il invoquait sept fois l'étoile de l'année (une des sept de la Petite Ourse) afin d'éloigner les calamités, puis, il saluait le Ciel, la Terre et les quatre points cardinaux. Enfin, l'empereur s'inclinait dans la direction des tombeaux de ses père et mère, puis retournait dans son appartement où lui étaient servies trois coupes de saké dans lequel on avait dissous des substances prophylactiques. Ensuite, depuis le pavillon de Pureté et de Fraîcheur, Seiryôden, où il logeait, l'empereur recevait, le temps d'une cérémonie appelée «Petite révérence de la cour », Kojôhai, l'hommage de ses serviteurs, hauts dignitaires et fonctionnaires, alignés dans le jardin. Enfin, l'empereur gratifiait ses 
serviteurs d'un banquet appelé "Réunion du premier jour » sechi-e, servi dans le Pavillon du sud, Nanden (également appelé Shishinden, Palais impérial). À cette occasion on présentait à l'empereur le calendrier de l'année qu'il promulguait en tant que maître du temps. Les fonctionnaires qui avaient le privilège de monter à la salle d'audience où se trouvait l'empereur, se tenaient au pied du pavillon, dans la cour, de chaque côté de l'escalier central. Les officiers des gardes de la section de gauche encadraient la cour qui se trouvait devant le pavillon à l'est et ceux de la section de droite celle qui se trouvait à l'ouest. Pendant le banquet, l'empereur était servi le premier. Il lui était présenté un grand nombre de plats différents. Les hauts dignitaires ne recevaient qu'une dizaine de mets et les autres fonctionnaires, installés dans la cour, qu'un nombre encore plus restreint de plats. Après le troisième service de saké, alors que l'office de la Couture dressait les coffres à gratifications, l'office de la Musique jouait, les musiciens se tenant debout dans la cour, tachigaku. L'assistance se regroupait ensuite dans la cour, debout en ordre hiérarchique, pour entendre la lecture d'un édit impérial et recevoir les gratifications.

Le deuxième jour de la première lune avaient lieu deux banquets, offerts par l'impératrice et le prince héritier, Nigĥu no daikyô. Les hauts dignitaires et fonctionnaires, alignés hiérarchiquement, saluaient l'impératrice d'une grande révérence, depuis le jardin du lieu où elle résidait, puis le banquet leur était servi.

Dans les premiers jours de l'année, si l'empereur avait encore son père, il lui rendait une visite appelée Chôkin gyôkô. Il pouvait également aller voir sa mère. L'empereur retiré accueillait son fils au pied des marches de son palais et le raccompagnait de la même façon ${ }^{18}$. Ces visites comportaient des banquets, des divertissements et des gratifications pour les hauts dignitaires qui accompagnaient l'empereur. Nous disposons d'un document extrêmement précieux pour nous représenter le spectacle qu'offraient ces célébrations : le Rouleau enluminé du cycle annuel des célébrations, Nenjû gyôji-emaki ${ }^{19}$. Dans celui-ci est illustrée une séance de danse donnée à l'occasion de la visite que fit à son père l'empereur Goshirakawa (1127-1192). La scène se déroule dans la cour et le jardin, au sud du pavillon principal. On devine, derrière les stores baissés, lcs dames de la cour ct l'cmpcrcur qui regardent le spectaclc. Sur la galerie qui entoure le pavillon, de hauts dignitaires sont assis, face au jardin. Vêtus de leur costume de cour sombre, ils tiennent leur insigne de dignité et laissent pendre leur traîne par dessus la rampe de la galerie. Dans la cour, deux longues nattes ont été placées de chaque côté des degrés du pavillon, sur lesquelles des dignitaires sont assis, les jambes croisées, leurs chaussures soigneusement posées devant eux. Certains tiennent à la main leur insigne de dignité, d'autres un éventail ou encore un arc. Au milieu de la cour s'exécute un danseur vêtu d'un pourpoint rouge pourvu d'une traîne, d'un pantalon blanc et de bottes noires. Il porte également un gilet orné de fourrure. Dans sa main gauche, dressée, il tient une baguette de tambour, sa main droite est posée sur sa hanche. Parallèlement aux rampes de l'escalier, de chaque côté du danseur, des fonctionnaires de la garde font une haie, assis sur des tabourets pliants recouverts d'une peau de tigre. Ils sont chaussés de bottes et vêtus de leur costume de cour sur lequel ils 
portent un carquois. Dans le jardin, des pruniers sont en fleur, et sur l'étang, quatre jeunes garçons rament, debout sur un bateau peint de couleurs vives, tandis que près d'eux barbotent des canards.

Le huitième jour, commençait la célébration bouddhique du palais, Gosai-e, au cours de laquelle des moines faisaient la lecture du sûtra appelé « Sûtra de la Radiance d'or, roi suprême entre les sûtra », Konkômyô saishô. ôkyô. Cette célébration avait lieu au Daigokuden, pavillon de l'Ultime Suprême, où se déroulaient les lectures ordinaires et extraordinaires de sûtra. Elle était considérée comme étant efficace pour assurer la protection du pays. Pour se rendre au pavillon de l'Ultime Suprême, les moines, pénétrant par les portes du Bonheur de l'est, Tôfuku. mon et du Principe Yang éternel, Eiyô. mon, défilaient dans la cour située au sud du pavillon ${ }^{20}$. Cette procession devait constituer un événement spectaculaire si l'on en croit le traitement dont il fait l'objet dans le Rouleau enluminé du cycle annuel des célébrations : les moines, dont certains portent à la main un brûle-encens pourvu d'un manche, défilent en rang dans la cour ${ }^{21}$, vêtus certains d'une robe jaune et d'autres d'une robe verte. La célébration bouddhique du palais, qui durait sept jours, donnait lieu, le dernier soir, à un spectacle de danse qu'illustre le Rouleau enluminé du cycle annuel des célébrations. Dans la cour sud du pavillon de l'Ultime Suprême douze bottes d'épis de riz, offertes par la province de Yamashiro, ont été posées sur le sol, en deux files de six, servant ainsi en quelque sorte à délimiter à l'est et à l'ouest l'espace de danse. En retrait de celui-ci, des fauteuils ont été installés sur lesquels sont assis des fonctionnaires de la garde. Au centre, quatre danseurs s'exécutent, portant d'amples costumes dont la manche droite du vêtement de dessus n'a pas été enfilée et des couvre-chefs au bonnet rouge vif et aux bords découpés retroussés. Au sommet des trois escaliers de la façade sud du pavillon, des subalternes portent des torches afin d'éclairer le spectacle. Des spectateurs sont assis, éparpillés dans la cour, tandis que dans le pavillon s'achèvent les célébrations auxquelles assistent quelques hauts dignitaires (que les rideaux à demi relevés laissent deviner), ainsi que des moines, assis au coin ouest de l'édifice. Un moine ne peut s'empêcher de se laisser distraire par les danseurs et tourne son visage vers le spectacle ${ }^{22}$.

Le seizième jour de la première lune se déroulait un banquet à la cour, au pavillon du sud. Pendant que le repas était servi aux convives, des femmes frappaient le sol de leur pieds en chantant, tôka, pour en réveiller les énergies, et faisaient des rondes autour des bâtiments du palais intérieur. Cette pratique fort ancienne, caractéristique d'une société agraire, est illustrée dans le Rouleau enluminé du cycle annuel des célébrations ${ }^{23}$ : dans la cour, au pied de l'escalier du palais impérial, des nattes sont disposées de manière à former un circuit carré relié au pavillon de la bibliothèque ; onze femmes, vêtues du costume complet à la chinoise, Kara shôzoku, la chevelure en partie relevée, le front orné d'un peigne décoratif, défilent, un éventail en bois de santal à la main droite et des feuilles de papier pliées aux couleurs dégradées à la main gauche, tandis que d'autres se préparent à entrer en scène. (Elles devaient effectuer trois fois le tour du circuit.) Des femmes, employées de la chancellerie privée et de la salle des danses féminines, veillent au bon déroulement de la cérémonie. Deux hauts 
dignitaires, assis sur une natte à l'abri de la galerie du palais, contemplent le spectacle. Le cerisier et le mandarinier, plantés de chaque côté de l'escalier, participent de leurs branches aux formes tortueuses à la beauté du décor.

Le dix-huitième jour, une séance de tir à l'arc avec prix, noriyumi, était organisée par les deux sections de la garde du corps. Les tireurs qui avaient été sélectionnés et classés préalablement formaient des paires entre section de gauche et de droite. Chaque paire tirait trois ou quatre flèches. La victoire revenait à celui qui avait le plus grand nombre de flèches près du but. Cette séance se déroulait dans la cour du palais impérial ${ }^{24}$, devant le pavillon du champ de tir à l'arc, ibadono, où s'installait l'empereur.

Le vingtième jour de la première lune, un banquet dit « du Palais intérieur », Nai. en, était donné par l'empcrcur au pavillon de la Bienveillance et du Bonheur, Jijuden. À cette occasion, l'empereur invitait ses proches serviteurs et des lettrés et l'on composait de la poésie en chinois. Dans la cour, à l'est du pavillon, une estrade était dressée sur laquelle des danseuses se produisaient à la nuit tombée. Nous pouvons imaginer ce spectacle en nous reportant à l'illustration qu'en propose le Rouleau enluminé du cycle annuel des célébrations : quatre danseuses, vêtues de somptueuses robes de couleurs vives et coiffées de diadèmes, dansent à la lueur des torches. Au premier plan, à droite, un prunier couvert de fleurs roses semble prendre part au spectacle ${ }^{25}$.

Au cours de la première lune, le principal ministre - parfois d'autres ministres également - donnait un grand banquet, Daikyô. Quand l'invité d'honneur arrivait, le ministre descendait l'accueillir dans la cour, au bas du degré. La plupart des hauts dignitaires et fonctionnaires étaient présents, selon leur rang, qui au pavillon principal, qui dans le jardin. Les subalternes n'étaient appelés qu'au milieu du banquet pour recevoir leur part de régal et de saké dans le jardin. La musique se faisait entendre et des gratifications étaient distribuées à toute l'assistance, en commençant par les hommes du rang le plus bas jusqu'aux hôtes principaux qui recevaient des chevaux ${ }^{26}$. Des banquets « exceptionnels " pouvaient avoir lieu à d'autres moments de l'année, par exemple pour inaugurer une nouvelle période par un acte faste ${ }^{27}$. Ces célébrations se déroulaient souvent en fin de journée ; il s'agissait là d'une occasion pour écouter de la musique et regarder des danses qui étaient exécutées sur une estrade dans la cour devant le pavillon du sud. Quand la nuit tombait, on allumait des flambeaux, qu'on éteignait quand la lune brillait. Tout comme la clarté de la lune, une légère brise, mêlant son murmure au son des instruments, rehaussait le plaisir du spectacle.

Au début de la deuxième lune ${ }^{28}$, on célèbrait la fête du sanctuaire Kasuga à Nara. La célébration consistait en l'envoi de messagers, un pour l'empereur, un pour l'impératrice et un pour le prince héritier. La veille de l'envoi, l'empereur prenait un bain et saluait les offrandes. Il s'installait ensuite dans la galerie orientale du palais de Pureté et de Fraîcheur, Seiryôden, et recevait son envoyé. Il assistait ensuite au spectacle que les danseurs exécutaient sur l'estrade située dans le jardin à l'ouest du Jijuden. On amenait ensuite les chevaux qui allaient être présentés aux divinités. Du 
saké était offert à l'assistance. L'envoyé recevait des gratifications et un ornement de tête, qu'il mettait pour aller faire la grande révérence dansante dans le jardin, tandis que les musiciens et danseurs recevaient eux aussi des gratifications.

Le troisième jour de la troisième lune pouvait avoir lieu un banquet auprès d'un cours d'eau sinueux, gokusui no en. Cette cérémonie, sur laquelle nous reviendrons plus en détail dans la suite de cet article, donnait lieu à des compositions poétiques en chinois et, plus exceptionnellement, en japonais.

Le même jour avaient lieu des combats de coqs, tori awase ou tôkei ${ }^{29}$. D'origine chinoise, les combats de coqs furent, semble-t-il, pratiqués au Japon dès le cinquième siècle ${ }^{30}$. Ces combats avaient lieu, pour ce qui est du palais impérial, dans la cour sud du Seiryôden, ou devant le pavillon de l'Expansion du Beau, Kokiden. Quand ces combats avaient lieu dans la résidence d'un dignitaire, ils se déroulaient alors dans le jardin. Le Rouleau enluminé du cycle annuel des célébrations propose une illustration très vivante de ces combats de coqs, à la fois chez le petit peuple ${ }^{31}$ et chez un dignitaire ${ }^{32}$. Dans le jardin, au sud de la résidence du dignitaire ont été installées, à la limite est et ouest du terrain de combat, deux tentes rayées de cinq couleurs, une pour l'équipe de droite, l'autre pour l'équipe de gauche, où les participants se préparent. On a par ailleurs planté dans la cour un pin qui symbolise l'équipe de droite et un saule pour l'équipe de gauche. À chacun de ces arbustes est attaché un jeune coq. Au milieu de la cour, deux coqs s'affrontent, encouragés par les cris d'un entraîneur. Le maître des lieux contemple le spectacle en compagnie de ses hôtes masculins depuis la galerie du pavillon. Trois femmes assistent au spectacle en épiant à travers les stores de bambou tressé qui sont baissés. Deux dames de compagnie, assises sur la galerie de communication, prennent part au spectacle, l'une se cachant le visage avec son éventail, l'autre avec sa manche. Des employés de la garde, vêtus de vêtements dits " de chasse » (utilisés dans la vie quotidienne et lors d'exercices), coiffés de leur bonnet noir, assistent au spectacle, assis à même le sol. Dans le jardin, derrière la loge dite " de droite ", des coqs attendent leur tour de combattre, couverts chacun, pour qu'ils ne s'échappent pas, d'une cloche en bambou tressé. À l'issue du combat, l'équipe gagnante poussait un cri et exécutait une danse. On distingue sur le rouleau enluminé, dans la tente de gauche, deux gardes qui aident un jeune garçon à se préparer pour danser.

Durant la quatrième lune avait lieu la fête des deux sanctuaires de Kamo au nord de la capitale, dédiés à des divinités du lieu auxquelles la cour rendait un culte tout particulier. Comme dans le cas de la fête de Kasuga à la deuxième lune, un fils de haut dignitaire était désigné comme messager impérial et un programme musical et chorégraphique était préparé. Après s'être acquitté de différentes célébrations, le cortège revenait dans la nuit au palais où l'on avait allumé pour l'occasion un feu dans la cour à l'est du pavillon de Pureté et de Fraîcheur. À sa lueur, des membres du corps de garde interprétaient une danse évoquant un épisode relatif à la divinité et destiné à la faire venir jouir du spectacle - qui était, semble-t-il, fort apprécié - des membres de la cour. Une collation et du saké étaient servis aux participants. 
Le cinquième jour de la cinquième lune, au cours d'une célébration dite « du cinquième début », tango no sechi. e, l'empereur assistait depuis le pavillon de la Vertu guerrière, Butokuden, à des courses de chevaux et à un concours de tir à l'arc monté qui se déroulaient dans la cour située devant le pavillon. Pour l'occasion, les fonctionnaires participants portaient tous des acores à leur coiffure, plante à laquelle on attribuait des vertus apotropaïques.

À la fin de la septième lune se déroulaient les luttes, sumai. Ces luttes, au cours desquelles s'affrontaient des paires d'hommes très forts recrutés dans les provinces, se déroulaient généralement dans la cour du pavillon du sud. Elles avaient à l'origine un sens religieux : il s'agissait de susciter les énergies du sol par le déploiement de forces humaines au moment crucial de la maturation du riz. Ces luttes servaient également à établir des pronostics pour l'avenir.

Dans la dernière nuit de l'année, un rite d'exorcisme était pratiqué. Pendant que l'empereur restait dans ses appartements où une cérémonie était célébrée pour lui, des hommes des gardes jouaient le rôle de démons et étaient expulsés, ce qui donnait lieu au palais à une grande animation : flambeaux, courses autour des pavillons et tirs de flèches se déroulaient dans le jardin.

En dehors des cérémonies « officielles », étaient organisées tout au long de l'année des célébrations exceptionnelles, qui pouvaient se dérouler - totalement ou en partie dans le jardin. Ainsi, dans ses notes journalières, Fujiwara no Michinaga décrit-il, à la date du 11 juin 1004, une cérémonie des Huit Leçons (au cours de laquelle on lisait les huit fascicules du sûtra du Lotus de la Bonne Loi), qui se déroula chez lui, à Tsuchimikado :

On sonne la cloche et on pénètre à la chapelle. Après que le moine maître de prédication est monté au siège élevé, on dresse les offrandes. Les moines descendent par deux escaliers, les princes et hauts dignitaires descendent du pavillon de l'Ouest. Pendant qu'ils s'alignent, des voix s'élèvent d'un bateau de chanteurs qui est au sud de la chapelle. Un bateau qui, de la même façon, glisse au pied du couloir, apparaît portant deux hommes habillés en boddhisattvas battant du tambour. Puis on processionne par trois fois autour de l'île centrale. Ensuite on remonte (à la chapelle) par le double degré. Les grands et les courtisans placent les offrandes devant le Bouddha, les hommes du cinquième rang les placent dans le jardin. Pendant ce temps, les bateaux de la musique viennent au lieu où se trouve le pin, l'estrade de danse avance entre les deux bateaux. Cependant, huit jeunes danseurs, tenant des fleurs à offrir au Bouddha, arrivent au bas du degré ; huit moines prennent les fleurs et les offrent au Bouddha. Ces jeunes danseurs se retirent en dansant la danse de l'Oiseau ${ }^{33}$. [...] Une fois qu'ils ont fait un tour de l'île, les bateaux repartent en direction de l'est, où ils se séparent, les voix s'entendent encore un moment $[\ldots]^{34}$.

En lisant cette description, on ne peut qu'être surpris du rôle que joue le jardin, et son étang en particulier, dans la mise en scène de cette célébration bouddhique. Précisons 
tout de suite que cette mise en scène et les effets surprenants qu'elle mettait en œuvre n'étaient rendus possibles que par l'architecture de la résidence de Michinaga. Une configuration spatiale différente aurait induit une organisation de la cérémonie distincte. Les organisateurs de cette célébration ont clairement cherché à tirer tout le parti qu'ils pouvaient de l'espace, afin de conférer à la cérémonie bouddhique une atmosphère céleste, surnaturelle.

Les notes journalières de Fujiwara no Sukefusa (1007-1057) relatent un fait survenu en Chôryaku 2, c'est-à-dire en 1038 : un bœuf est mort dans l'enceinte du palais qui s'en trouve souillé ${ }^{35}$. L'empereur Gosuzaku ordonne alors que l'on prépare le jardin (sans doute que l'on installe des nattes et un mobilier sommaire) pour qu'il puisse annoncer la souillure aux divinités et prier. L'empereur priera dans le jardin trois nuits de suite. C'est encore sur le sol du jardin que l'empereur ira prier quand, au cours de la première année de Chôkyû (1040), le sanctuaire de Toyo. uke s'est effondré, et que la divinité a fait savoir par le truchement de la vestale son refus de recevoir le messager impérial. On a étalé à même le sol plusieurs nattes, dressé tout autour des paravents, et placé un flambeau. L'empereur se dirige d'abord vers le lieu du jardin d'où il doit saluer les divinités - un chemin de nattes a été établi à cet effet - puis entre dans l'espace délimité par les paravents pour lire son vœu, qu'il a écrit lui-même. La séance dure trois heures, de neuf heures et demie à onze heures et demie du soir. Ces dévotions pouvaient se poursuivre sur plusieurs jours.

Il ressort de la description de ces célébrations - tout particulièrement dans le cas des cérémonies religieuses -, que le jardin n'était pas seulement un espace qui permettait de s'ébattre plus à son aise, c'était aussi (et avant tout ?) le lieu où le contact avec les divinités pouvait se faire le plus directement. Pour communiquer avec les dieux, la terre, les arbres, les fleurs, les pierres, l'eau et le vent constituaient sans doute un environnement plus propice que l'espace clos, aussi imposant soit-il, du palais et des résidences.

\section{Le jardin comme espace ludique}

La description des différentes cérémonies qui se déroulaient dans le jardin ne doit pas nous faire occulter le fait qu'à l'époque de Heian le jardin est également un lieu où l'on s'amuse. Nous avons mentionné au début de cet article le fait que certains jardins de l'époque de Heian étaient bâtis de manière à évoquer certains paysages célèbres. Nous citions l'exemple du jardin de Minamoto no Tôru, dans lequel avait été reproduit la baie de Shiogama. Le souci du détail avait été poussé jusqu'à faire transporter à grands frais de l'eau de mer pour en alimenter l'étang et permettre ainsi de procéder à l'extraction du sel, de manière à reproduire sur place l'activité principale des riverains de Shiogama ${ }^{36}$. Est-il besoin de préciser que toutes ces activités n'avaient qu'un but : le plaisir, un plaisir sans doute similaire à celui que devait éprouver Marie-Antoinette quand elle jouait à la bergère dans son Hameau de Trianon. Si l'excentricité de Tôru est restée fameuse dans l'histoire, son goût pour les lieux animés de figurants ne fut sans 
doute pas unique : l'Ima kagami, Miroir du temps présent, récit historique achevé en 1070, ne nous dit-il pas que Tachibana no Toshitsuna (1028-1094), l'auteur présumé du Sakutei-ki, construisit dans le jardin de sa résidence de Fushimi un paysage de montagne dont il faisait, à l'occasion, parcourir les sentiers par de faux voyageurs ${ }^{37}$ ? Aussi peut-on se plaire à voir dans les jardins de l'époque de Heian les précurseurs de nos parcs d'attractions...

Les Japonais de l'époque de Heian pratiquaient également un jeu de ballon appelé kemari, ce qui signifie «balle au pied ». On délimitait pour ce jeu un terrain dans le jardin, à l'écart des bâtiments : quatre arbres d'essences différentes, espacés de six ou sept mètres, étaient plantés en carré : un pin, un prunier, un saule et un cerisier (arbres qui pouvaient être remplacés par des repères). Les arbres-repères devaient mesurer au moins quatre mètres cinquante - hauteur à laquelle devait être envoyée la balle - et leurs branches les plus basses se trouver un peu au dessus du niveau de la coiffure des joueurs qui étaient vêtus du "vêtement de chasse », utilisé pour l'exercice, et coiffés d'un bonnet. Le terrain était débarrassé de ses cailloux et aplani à l'aide de sel et de sable. (On le protégeait après le jeu en le couvrant de nattes.) Les participants étaient répartis en trois catégories : joueurs, assistants et arbitres. Au nombre de huit, les joueurs se répartissaient par paires au pied de chaque arbre. Les assistants (un par joueur) étaient chargés de ramener les balles égarées. Les arbitres, choisis parmi des personnes d'âge plus mûr, veillaient au bon déroulement de la partie et comptaient les points. Leur nombre n'était pas fixe. Le ballon, fait de peau de daim, était, en prévision d'une partie, suspendu à l'aide de cordelettes de papier torsadé à une branche du pin ou du saule. La partie commençait par l'envoi de la balle, honneur qui revenait à l'un des joueurs. Le jeu consistait à recevoir et renvoyer la balle, à l'aide exclusivement des pieds, sans la laisser tomber sur le sol : la correction, l'élégance des déplacements sur le terrain, étaient pris en compte pour le calcul des points. On commençait à compter les points après cinquante passes du ballon. Si la partie n'avait pas été interrompue par la chute du ballon (ou la tombée de la nuit), la victoire était attribuée à mille points. Ce jeu, dont la première exécution de l'année pouvait donner lieu à une célébration au début du troisième mois, est illustré de manière très vivante dans le Rouleau enluminé $d u$ cycle annuel des célébrations ${ }^{38}$. La scène se situe dans le jardin d'une résidence. Les joueurs, vêtus d'habits de différentes couleurs - certains tiennent un éventail à la main -, suivent des yeux la balle qui s'élève entre les arbres en fleurs. L'arbitre (?), assis négligemment sur les degrés de la demeure, lit un document tandis qu'une dame, que la partie ne semble pas intéresser, est installée sur la galerie, absorbée dans la contemplation d'un cerisier en fleurs. Un peu à l'écart du terrain de jeu, un jeune page rechausse l'un des joueurs. Dans un coin, un spectateur accroupi, également vêtu de son « habit de chasse », assiste, fasciné, à la partie.

Si l'on en croit le nombre de leurs occurrences dans les textes de l'époque de Heian, les promenades en barque sur l'étang du jardin constituaient l'un des plaisirs favoris de l'aristocratie, les hauts dignitaires ne dédaignant pas à l'occasion de prendre eux- 
mêmes les rames. Ainsi dans son Journal, qu'elle tînt pour les années 1008-1009, Murasaki Shikibu décrit une partie de canotage qui eut lieu à la cour :

La nuit du lendemain, la lune luit splendide, et comme la saison elle-même est plaisante, les jeunes personnes s'amusent à monter en barque. Plus qu'aux temps où elles portent des couleurs diverses, les silhouettes pareillement vêtues de blanc et les chevelures se détachent nettes et sans défaut. Kodaïfu, Genshikibu, Miyagi no Jijû [...] et d'autres sont assises près du rebord ; le Conseiller Commandant de la Gauche et le sire Commandant, fils de Monseigneur, les invitent, et le Conseiller Commandant de la Droite Kanétaka, maniant la perche, les font embarquer. Une partie d'entre elles se dérobent, mais envieuses sans doute malgré tout, elles restent à regarder. Sur le sable blanc du jardin, les silhouettes et les visages reflétant le clair de lune ont plaisante apparence ${ }^{39}$.

Les embarcations utilisées pouvaient être des bateaux de fête, qui allaient généralement par paires. L'un avait la proue ornée d'une tête de dragon, l'autre de phénix ${ }^{40}$. Ces bateaux étaient assez larges et à fond plat afin de permettre que des musiciens et des danseurs s'y installent, car des spectacles étaient parfois donnés sur l'eau. La magie de telles représentations peut être perçue à la lecture de la description qu'en fait Murasaki Shikibu, dans son Journal :

À la tombée de la nuit, la musique retentit, enchanteresse. [...] Après les danses des Dix mille années, de la Grande Paix, du Palais Fortuné, l'orchestre exécute le Chôkeishi pour clore le concert, et quand les barques contournent le promontoire de la colline de l'étang, à mesure qu'elles s'éloignent, le son de la flûte, le bruit des tambours et le vent dans les pins se confondent dans l'épaisseur des arbres en une superbe harmonie ${ }^{41}$.

Les promenades en barque sur l'étang du jardin au son des instruments de musique n'ont pas seulement diverti les gens de la cour; elles ont également inspiré des pages parmi les plus belles de la littérature japonaise. Il suffit pour s'en convaincre de lire la description sur laquelle s'ouvre le livre vingt-quatrième du célèbre Dit du Genji de Murasaki Shikibu, intitulé Les Papillons ${ }^{42}$.

Étroitement liés aux jardins dont ils constituent en quelque sorte la mise en abyme, se trouvent les œuvres crées dans le cadre du jeu appelé « concours de jardins », senzai awase, apparu à l'époque de Heian. Ce jeu est une variante des concours d'objets, mono awase, divertissement d'origine chinoise. Deux équipes dites « de gauche » et «de droite », rivalisaient pour présenter l'objet le plus rare ou le plus précieux. Ces objets, définis à l'avance, pouvaient être des racines d'iris, des coquillages, des parfums, des éventails, etc. Dans le cadre des senzai awase il s'agissait pour chacune des équipes de créer le plus beau jardin. Le jardin pouvait être planté dans une cour, ou bien présenté sur un support. Dans l'Eiga monogatari, Récit de la splendeur Ides Fujiwara], chronique de l'époque de Heian, est décrit un senzai awase qui eut lieu le 15 de la huitième lune, en Kôhô 3 (966) ${ }^{43}$. Devant le Seiryôden, la résidence de l'empereur, on répartit les concurrents en deux équipes. Le chef de l'équipe de gauche 
était l'intendant de l'atelier des peintures, $e$-dokoro (Fujiwara no Naritoki) et celui de l'équipe de droite, l'intendant de l'atelier des fabrications, tsukumo-dokoro (Fujiwara no Tamemitsu). Les ateliers des fabrications et des peintures avaient pour charge de fabriquer la plupart des objets utilisés à la cour ${ }^{44}$; aussi chacune des équipes s'efforçat-elle de mettre en valeur son savoir-faire. Dans le cas de ce concours, les deux jardins furent présentés sur des supports :

L'équipe de l'atelier des peintures avait peint un plateau au contour découpé et, sur celui-ci, représenté toutes sortes de fleurs plus belles que nature, un cours d'eau et de grands rochers, puis, à l'aide [de fils] d'argent, elle avait fabriqué un enclos treillagé dans lequel avaient été placés divers insectes vivants. Elle avait [également] peint une promenade [le long de la] rivière Ôi, et sur un bateau pour la pêche au cormoran, des torches allumées ; à côté des insectes, on avait écrit un poème. L'équipe de l'atelier des fabrications avait sculpté [sur le plateau] une plage au rivage découpé, originale, lui donnant l'aspect qu'elle aurait à marée haute, planté toutes sortes de fleurs artificielles, sculpté et joint des pins et des bambous ; c'était très original. Le poème était fixé à une fleur de pratinie.

Chacun de ces jardins était, afin d'en rehausser l'originalité, accompagné d'un poème. Le poème de l'équipe de gauche qui, rappelons-le, avait placé des insectes vivants sur son dessin, exprime le vœu de longévité destiné à l'empereur :

Kimi ga tame

Hana uhesomu to

Tsugenedomo

Chiyo matsu mushi no

Ne ni zo nakinuru
Bien que nous ne disions pas

Que c'est pour vous Sire

Que nous commençons à planter ces fleurs

Espérant vos mille automnes,

Les grillons, par leur chant, vous l'expriment ${ }^{45}$.

Le poème de l'équipe de droite était, lui, fixé à une fleur de pratinie artificielle :

Kokoro shite

Kotoshi ha nihohe

Wominaheshi

Sakanu hana zo to

Hito ha miru to mo
Prends bien soin

D'embaumer cette année

Pratinie !

Même si les gens s'aperçoivent

Que tu es une fleur qui point ne s'épanouit.

Le trait d'esprit de ce poème réside dans le fait qu'on demande à une fleur artificielle d'embaumer. Le Eiga monogatari ne nous dit pas quelle équipe remporta la victoire (fut-elle seulement attribuée ?). Dans ce concours, qui se déroula dans la cour devant le palais, le jardin réel est totalement absent (le concours aurait pu aussi bien se tenir dans une salle); le jardin n'existe ici que par sa représentation. Les participants semblent s'être efforcés dans ce concours - peut-être pour mettre en valeur le talent des ateliers des peintures et des fabrications - de créer de toutes pièces un jardin idéal, mais $\operatorname{artificiel}^{46}$. 


\section{Le jardin comme espace de composition poétique}

Revenons à présent plus en détail sur les « banquets au bord d'un cours d'eau sinueux » gokusui no en, que nous nous sommes contenté de mentionner dans la partie de notre article consacrée aux célébrations. Comme nombre de pratiques lettrées adoptées au Japon, ces banquets au bord de l'eau sont d'origine chinoise ${ }^{47}$. Le banquet était célébré sur les rives d'un cours d'eau sinueux, naturel ou artificiel, et comportait des joutes de compositions poétiques, traditionnellement en chinois. Les convives étaient placés sur une natte à quelque distance les uns des autres, sur chaque rive du ruisseau. Des coupes d'alcool sur des supports flottants en forme d'oiseaux étaient lâchés sur le cours d'eau, en amont des convives ${ }^{48}$. Les participants devaient composer leur poème avant le passage de la coupe devant eux. S'ils avaient réussi, ils pouvaient boire le contenu de la coupe. Il semble que cette pratique, fort ancienne, ait été à l'origine un rite de purification, elle se déroulait le troisième jour du troisième mois. $\mathrm{Si}$ l'on en croit le Nihon shoki, Annales du Japon (ouvrage achevé en 720), ces banquets furent pratiqués au Japon dès le $\mathrm{v}^{\mathbf{e}}$ siècle ${ }^{49}$; ils ne sont toutefois attestés qu'au VIII ${ }^{\mathbf{e}}$, et n'étaient célébrés qu'exceptionnellement au Japon ${ }^{50}$. L'exemple sur lequel nous allons nous pencher à présent est une variante de ce type de manifestation; il s'agissait cette fois de produire non pas des poèmes chinois, mais des waka, des poèmes de trente et une syllabes en pur japonais ${ }^{51}$. Le Ki no shishô gokusui no en waka ${ }^{52}$ ou Poèmes japonais [composés au cours] d'un banquet au bord d'un cours d'eau sinueux [organisé] par maître Ki no [Tsurayuki] serait le plus ancien exemple qui nous ait été conservé de ce type de rencontre poétique ${ }^{53}$. Huit poètes participèrent à cette manifestation, qui se déroula vraisemblablement le 3 du troisième mois dans le jardin de la demeure de l'organisateur, Ki no Tsurayuki : Ôshikôchi no Mitsune (actif à la fin du $\mathrm{IX}^{\mathrm{c}}$ siècle et dans la première moitié du $\mathrm{X}^{\mathrm{e}}$ ), Fujiwara no Korehira (876-938), Ki no Tomonori (?-905 ?), Fujiwara no Okikaze (actif au début du $x^{e}$ siècle), Ôe no Chisato (actif fin $I X^{\mathrm{e}}$, début $\mathrm{x}^{\mathrm{e}}$ siècle), Sakano.ue no Korenori (actif début $\mathrm{X}^{\mathrm{e}}$ siècle), Mibu no Tadamine (actif dans la première moitié du $x^{e}$ siècle) et l'organisateur, Ki no Tsurayuki (?-945). Les huit participants composèrent un waka sur chacun des trois sujets imposés. Ceux-ci se présentent comme des vers extraits de poèmes chinois mais sont en fait des œuvres originales composées au Japon ${ }^{54}$ : « Des fleurs de cerisier flottent sur les eaux printanières ", "Clarté des torchères placées au bord de l'eau », «La lune pénètre dans un rapide [couvert] de fleurs; obscurité 55 ". Nous pouvons déduire de ces thèmes et des poèmes qu'ils inspirèrent que ce gokusui no en s'est déroulé la nuit, à la lumière de torchères. Le fait qu'il s'agisse d'une manifestation qui se déroulait au bord de l'eau le 3 du troisième mois explique la récurrence des deux éléments que sont l'eau et les fleurs de cerisier, dont c'est la saison. Des minutieux recoupements opérés par Hashimoto Fumio pour déterminer en quelle année les huit poètes pouvaient se trouver ensemble à la capitale, il ressort que cette manifestation se tint probablement en Engi 3, c'est-à-dire en $903^{56}$. Examinons à présent, pour chacun des sujets présentés, quelques poèmes, afin de saisir l'atmosphère de la manifestation. 
Le sujet « Des fleurs de cerisier flottent sur les eaux printanières » a donné lieu à divers traitements : certains poètes ont choisi conventionnellement de décrire les fleurs tombées que charrie le cours d'eau. D'autres ont traité le sujet de manière allusive. Tous, sans exception, ont introduit un élément qui ne faisait pas partie du sujet : le parfum des fleurs. Voyons à présent le poème de Mitsune qui a choisi un traitement allusif du sujet :

Yamigakure

Iwama wo wakete

Yuku mizu no

Kowe sahe hana no

Ka ni zo shimikeru
Même le bruit de l'onde

Qui se fraye un chemin entre les rochers

Plongés dans l'obscurité

Embaume du parfum

Des fleurs de cerisiers

C'est également un traitement allusif qu'a choisi Ôe no Chisato. Dans sa composition, le parfum trahit la présence de cerisiers, pour lors invisibles :
Haru no yo no
Dans la nuit de printemps
Sara yuku mizu no
On hume le parfum
Ka wo kageba
De l'onde qui va son cours :
Itsuku to mo naku
Il doit se trouver quelque part
Hana koso arikere
Des fleurs de cerisiers

Tadamine a choisi de chanter dans son poème le moment où le parfum des fleurs de cerisiers qui flottent à la surface de l'eau emplit peu à peu l'obscurité ; il composa pour cela un waka que l'on pourrait qualifier de baudelairien!
Iwa sosogu
Progresse avec l'eau
Mizu ni yukitsutsu
Qui vient battre les rochers
Hana no ka no
Le parfum des fleurs;
Sora no medetaki
C'est là pour le ciel
Yamigakure kana
D’auspicieuses ténèbres !

Le second sujet "Clarté des torchères placées au bord de l'eau » a inspiré des poèmes qui chantent l'éclat de ce mode d'éclairage dont les effets ont de toute évidence émerveillé les participants. Seul Mitsune déplore le grand nombre de torchères qui, par leur lumière, déflorent la magie, le mystère, du paysage :

Mizu soko no

Kage mo ukaberu

Kakaribi no

Amata ni miyuru

Haru no yohi kana
Même le paysage du fond de l'eau,

Fait remonter à la surface,

Le feu des torchères

Que l'on voit en grand nombre

En cette nuit de printemps !

Okikaze a quant à lui chanté l'image des reflets épars sur les vaguelettes, semblables à des perles ou des gouttes de pluie. Il a choisi par ailleurs de faire allusion à l'alcool que contenaient les coupes que l'on faisait flotter en employant le verbe chiru (que nous 
avons traduit ici par «se déverser » et qui signifie « couler, déborder »-d'une coupe par exemple) ${ }^{57}$.

Shira tama no

Fureru toya mimu

Migiwa yori

Sayakeku chireru

Kakaribi no kage
$\mathrm{Ne}$ dirait-on pas

Qu'il est tombé de blanches perles?

Depuis la rive

Se déverse, limpide,

Le reflet des torchères.

Le troisième sujet «La lune pénètre dans un rapide [couvert] de fleurs : obscurité » est plus complexe. L'expression " hana no se » signifie littéralement : "courant rapide de fleurs ». La signification de cette expression n'est pas claire. Le sens premier est, semble-t-il, le suivant : quand le reflet de la lune arrive à hauteur du rapide où l'eau est couverte de fleurs, il disparaît, et l'obscurité augmente. Certains poètes, sans doute mis en difficulté par le sujet, ont préféré le traiter de manière métaphorique ; «le courant rapide de fleurs » ne désigne alors que des arbres couverts de fleurs aux ramures ployantes semblables à des vagues. Curieusement, sur les huit poèmes composés, un seul contient l'image de l'eau - que pourtant sous-entendait l'expression " courant rapide »-, le poème de Korenori :

$\begin{array}{ll}\text { Hana nagasu } & \text { Je voulais voir } \\ \text { Se wo mo mirubeki } & \text { Le courant rapide qui charie les fleurs } \\ \text { Mikazuki no } & \text { Mais la lune du troisième jour } \\ \text { Warete irinuru } & \text { S'est en partie cachée } \\ \text { Yama no wochikata } & \text { Là-bas derrière la montagne. }\end{array}$

Ce traitement en apparence banal du sujet a permis à l'auteur de ce poème - qui sera au début du XIII ${ }^{e}$ siècle inclus dans la huitième anthologie impériale Shinkokin-wakash $\hat{u}$ (section : «Poèmes de printemps », $n^{\circ} 152$ ) - de jouer sur les homophonies afin de faire allusion aux coupes et à l'alcool qui étaient de rigueur lors d'une telle manifestation. La lune du troisième mois, mikazuki, permet de lire en filigrane les deux mots mika « fiole à saké » et tsuki « coupe »; le warete du quatrième vers que nous avons rendu par « en partie » signifie littéralement «brisé », et irinuru que nous avons traduit par « s'est cachée » signifie également « a pénétré », «a coulé » « sombré ». Kubota Jun lit dans ce poème une allusion à une coupe de saké qui se serait brisée et aurait sombré au fond de l'eau ${ }^{58}$.

Comment ne pas ne pas citer, pour clore cette partie, une des compositions de celui qui organisa la manifestation : Tsurayuki. Celui-ci, comme la plupart des autres poètes, a éludé l'image de l'eau, qui ne subsiste que dans l'emploi métaphorique de l'expression " rapide de fleurs ». Celle-ci désigne ici les vagues que forment les branches des cerisiers en fleurs sur le Mont Ogura (sis à Kyôto) dont le nom, par homophonie, évoque une "légère obscurité ». L'auteur a sans doute utilisé ce toponyme pour renforcer l'idée d'obscurité voulue par le sujet. 


$\begin{array}{ll}\text { Irinureba } & \text { Une fois [l'astre] couché } \\ \text { Wogura no yama no } & \text { Le [paysage] là-bas } \\ \text { Wochi ni koso } & \text { Sur le mont Ogura, légèrement obscur, } \\ \text { Tsuki naki hana no } & \text { Devient vraiment } \\ \text { Se tomo narinure } & \text { Un rapide de fleurs, sans lune }\end{array}$

Les poètes conviés à ce banquet ont - dans la mesure où cette rencontre s'est réellement tenue - utilisé le jardin comme lieu de composition et comme matériau. Les sujets contenaient des éléments qui devaient se trouver dans le jardin : les torchères, le cours d'eau, les fleurs de cerisier. Il est intéressant de noter que les auteurs ont produit des variations sur les thèmes du sujet, sans introduire d'élément véritablement descriptif. Une lecture, même attentive, des poèmes produits, ne révèle aucun élément concret de l'architecture du jardin. Plutôt que le paysage, c'est l'instant que les poètes se sont efforcés de traduire.

L'inventaire des activités qui se déroulaient dans les jardins à l'époque de Heian nous a permis de saisir l'importance de cet espace dans la vie quotidienne de l'aristocratie. Le jardin est alors un espace théâtralisé, un espace du rituel : c'est là qu'est mis en scène le pouvoir, se déroulent parades et défilés ; c'est là encore qu'ont lieu les célébrations les plus austères, mais également les plus festives et que, d'une ample révérence, le sujet exprime son respect au souverain. En tant qu'espace naturel, le jardin est également une sorte de point de jonction entre le monde des hommes et celui des dieux; c'est là que, accroupi sur le sol, un empereur a choisi de prier et que des femmes, battant la terre des pieds, tentent d'en éveiller les énergies. Le jardin est aussi - et surtout - à l'époque un espace où l'homme peut laisser libre cours à ses gestes et à ses émotions : c'est là qu'il danse, qu'il fait la fête, qu'il joue et qu'il crie. Les nombreuses descriptions conservées dans les textes témoignent du goût des Japonais pour les activités d'extérieur : dans le jardin, l'homme de ce temps se sent libre et joyeux. Espace ludique, espace cérémoniel, le jardin est également le théâtre de différentes expressions artistiques : danse, poésie, musique s'y répondent. Il préfigure en cela le jardin occidental qui, selon la belle définition de $\mathbf{J}$. Baltrusaitis, « est toujours le lieu d'une évasion vers l'Arcadie antique ou le paradis des premiers hommes, vers l'ermitage dans la solitude, vers le théâtre et vers la fête ${ }^{59}$ ».

\section{Notes}

1. Manière de montrer les jardins de Versailles par Louis XIV, Éditions de la Réunion des Musées nationaux, Paris, 1982, réédition 1992.

2. Préface à l'ouvrage cité en note 1, p. 6 .

3. L'ouvrage de référence sur le sujet est l'Histoire des jardins de la Renaissance à nos jours, publié sous la direction de Monique Mosser et Georges Teyssot, Flammarion, Paris, 1991.

4. Voir Michel Conan, Dictionnaire historique de l'art des jardins, Hazan, 1997, qui, à l'article Fête, cite en particulier la réception des ambassadeurs aux Tuileries ou la fête de Versailles du 18 juillet 1668. 
5. Pour un bref rappel de l'histoire des jardins japonais, des principes esthétiques, pratiques et religieux mis en œuvre, nous renvoyons à la préface de notre traduction du plus ancien traité qui nous ait été conservé (début du $\mathrm{XI}^{\mathrm{e}}$ siècle) : De la création des jardins - Traduction du Sakutei-ki, Monographies de la MFJ, série « Pensée japonaise », Maison franco-japonaise, Tôkyô, 1997.

6. On trouvera un plan d'ensemble de ce type de résidences dans l'introduction de notre traduction du Sakutei-ki, op. cit. p. 4. Les principes qui régissaient l'ordonnancement des jardins des résidences de la noblesse s'appliquaient également aux jardins des temples bouddhiques de la secte de la terre pure, jôdo shâ. Le palais impérial était quant à lui bâti selon un plan particulier que nous ne pouvons détailler ici : nous renvoyons à l'ouvrage de Francine Hérail, Notes journalières de Fujiwara no Michinaga, tome 1, Droz, Genève, 1987, p 109-129.

7. Les résidences de Fujiwara no Yorimichi et de Minamoto no Tôru, nobles de haut lignage, s'étendaient sur une surface d'un peu plus de quatre hectares, ce qui représentait un maximum pour une enceinte privée à l'intérieur de la capitale Heian, l'actuel Kyôto.

8. Le pavillon de pêche, construit comme toute la résidence sur pilotis, avançait au dessus de l'étang.

9. Sur ce jardin malheureusement disparu et le personnage excentrique qui le fit bâtir, voir l'étude minutieuse de Bernard Frank, Un jardin fameux de l'époque de Heian : le Kawara no in, Annuaire de l'École Pratique des Hautes Études, IV' section, Sciences historiques et philologiques, 1976-1977, p. 1035-1058.

10. La présence d'un cours d'eau ou d'une source était un élément primordial dans le choix d'un terrain ; l'eau n'était pas uniquement utilisée pour le jardin, elle l'était bien évidemment pour les besoins domestiques, et le ruisseau qui passait sous les corps de bâtiments, jouait le rôle d'évacuation des eaux usées.

11. De la création des jardins, op. cit., p. 48 -58.

12. Ibid, p. 20.

13. Cette partie de notre article doit beaucoup aux travaux de Francine Hérail, en particulier aux Notes journalières de Fujiwara no Michinaga, Droz, Genève, vol. 1, (1987), vol. 2 (1988), vol. 3 (1991) et à La Cour du Japon à l'époque de Heian aux $X^{\leftarrow}$ et XF siècles, Hachette, Paris, 1995.

14. La Cour du Japon à l'époque de Heian aux $X^{\mathrm{c}}$ et $X r^{\mathrm{c}}$ siècles, op. cit., p. 58.

15. Les cours sont, à l'époque de Heian, assimilées à des jardins. Le terme niwa désigne d'ailleurs dans la langue ancienne indifféremment un jardin ou une cour. Le terme senzai, qui désigne plus particulièrement la partie plantée et arborée d'un jardin, était également utilisé dans le sens de jardin. Il convient de remarquer que le palais intérieur, où résidait l'empereur, ne comprenait que des cours, dont certaines étaient ornées de quelques arbres ou végétaux. (Ainsi, par exemple, l'escalier conduisant au palais impérial, Shishinden, était-il flanqué à l'ouest d'un tachibana, citrus nobilis, et à l'est d'un cerisier.) Voir le plan du Palais Intérieur dans Francine Hérail, Notes journalières de Fujiwara no Michinaga, op. cit., p. 121.

16. Sakutei-ki, Sagami shobô, 1964.

17. Ibid., p. 76-77. 
18. Nous trouvons une illustration de ce rite dans le Rouleau enluminé du cycle annuel des célébrations, Nenjû gyôji-emaki, coll. « Nihon no emaki ", n 8, Chûôkôronsha, réed. 1990 , p. 6-7.

19. Voir la note précédente. Ce rouleau fut originellement peint à fin de l'époque de Heian, mais brûla avec le palais impérial au début de l'époque d'Edo (1603-1867). Il n'en subsiste malheureusement que des copies. Celle qui est reproduite dans l'ouvrage cité, considérée comme la meilleure, fut sans doute peinte dans la seconde moitié du $\mathrm{XVII}^{e}$ siècle. Pour la scène décrite voir les pages 10-11.

20. Il ne nous a pas été possible de localiser ces deux portes. N'ont-elles pas été rebaptisées?

21. Voir le Rouleau enluminé du cyle annuel des célébrations, p. 35.

22. Ibid., p. 38-39.

23. Ibid., p. 50.

24. Ibid. Une de ces séances est illustrée p. 22-23.

25. Ibid., p. 28-29.

26. Ibid. Ce banquet est représenté p. 30-31 et 50-51.

27. Par exemple quand l'empereur retournait dans son palais qui venait d'être reconstruit.

28. Cette célébration avait également lieu au début de la onzième lune.

29. Les combats de coqs pouvaient également avoir lieu à une date différente.

30. Voir les annales du Japon, Nihon shoki, à la septième année du règne de l'empereur Yûryaku (en 462), où il est écrit que l'empereur assista à un combat de coqs, coll. « Nihon koten bungaku taikei », vol. 67, tome 1, Éditions Iwanami, p. 472-474.

31. Le petit peuple assiste au combat de coqs dans la cour d'un sanctuaire.

32. Le Rouleau enluminé du cyle annuel des célébrations, op. cit., p. 14-19.

33. Francine Hérail, op. cit. (1987) p. 372-373, décrit l'argument de la danse dans les termes suivants : «Au monastère de Gi.on dans l'Inde un jour de célébration, un oiseau est venu, dont la grâce a inspiré la danse et dont le chant exprimait l'enseignement du Bouddha. Quatre jeunes garçons, portant des ailes, dansent en frappant des sortes de claquettes qui imitent le chant des oiseaux. »

34. Midô kanpaku-ki zen chûshaku, annoté par Yamanaka Yutaka, Takashina shoten, 1994, vol. 1, p. 99-104. La traduction citée est celle de Francine Hérail, op. cit. (1987) p. 371.

35. Nous remercions Mademoiselle Francine Hérail de nous avoir non seulement indiqué l'existence de ces célébrations, mais également de nous avoir fait tenir, pour les passages concernés, la traduction qu'elle prépare de ces notes journalières.

36. Voir Bernard Frank, op. cit., p. 1041.

37. Sakakibara et al., Ima kagami, honbun oyobi sôsakuin, Éditions Kazama-shoin, 1974, p. $100-102$.

38. Le Rouleau enluminé du cyle annuel des célébrations, op. cit., p. 18-19.

39. Murasaki Shikibu nikki, coll. "Shin nihon koten bungaku taikei ", vol. 24, Iwanami shoten, 1989, p. 269-270. Traduction René Sieffert, Publications Orientalistes de France, 1978, p. 22.

40. Murasaki Shikibu écrit dans son Journal, édition japonaise (op. cit., p. 274, traduction française p. 27) : «Au jour dit, Monseigneur fait approcher les barques nouvellement 
construites et les passe en revue. Les têtes de dragon et d'oiseau fantastique sont d'une facture splendide, si parfaites qu'on les imaginerait vivantes. »

41. Ibid., p. 31.

42. Voir Genji monogatari (chap. « Kochô »), coll. "Shin nihon koten bungaku taikei ", Éditions Iwanami, t. II, p. 400-401, traduction René Sieffert, Le Dit du Genji, POF, 1988 , t. 1, p. 485.

43. Eiga monogatari, coll. «Nihon koten bungaku taikei », vol. I, réed.1971, Éditions Iwanami, p. 50-51.

44. Voir Francine Hérail, Fonctions et fonctionnaires japonais au début du $x f^{e}$ siècle, Publications orientalistes de France, p. 214.

45. L'original japonais joue sur l'homophonie de matsu, « attendre " (que nous avons rendu par «espérer »), et matsumushi, «grillon ».

46. Un exemple de ce type de concours pour lequel des plantes naturelles ont été disposées dans la cour devant le palais de la princesse impériale Kishi eut lieu le huitième mois de la troisième année de Tenroku (972). Voir Kokon chomonjû, coll. « Shinchô nihon koten shûsei », Éditions Shinchôsha, vol. II, 1986, p. 337. On pourra également lire dans ce même receuil, au livre 19 intitulé Sô moku, Herbes et arbres, p. 336-337, une version plus laconique de notre concours.

47. Bien que cette pratique soit plus ancienne, le banquet au bord d'un cours d'eau sinueux que donna en 353 le célèbre écrivain et calligraphe chinois Wang Xizhi (321-379) dans son Pavillon des orchidées est resté fameux. On peut voir la reproduction d'une peinture chinoise illustrant ce banquet dans l'ouvrage d'Irmtraud SchaarschmidtRichter, Le Jardin japonais, Office du livre, Fribourg, 1979, p. 23.

48. Voir la photographie d'une reconstitution de cette cérémonie, telle qu'elle pouvait se dérouler à l'époque de Heian, dans l'ouvrage de Günter Nitschke, Le Jardin japonais, Éditions Benedikt Taschen, Cologne, 1993, p. 62-63.

49. Nihon shoki, Livre 15, Kenzô tennô, coll. « Nihon koten bungaku taikei », vol. 67, Éditions Iwanami, $6^{c}$ réed. 1972 , p. 120, 124. La mention des gokusui no en pourrait être un ajout du compilateur.

50. Selon Francine Hérail (op. cit., 1987, p. 75), les deux dernières séances de ce type au $\mathrm{X}^{\mathrm{e}}$ siècle eurent lieu en 958 et 966 , mais deux séances du même genre eurent lieu au début du $\mathrm{XI}^{\mathrm{c}}$ siècle, en 1005 et 1006 . Plus tard, sous le règne de l'empereur Go. ichijô, en 1018 et 1031 , la cérémonie fut ressuscitée.

51. Les trente et une syllabes du waka ou " poème japonais " sont réparties en cinq vers de 5/7/5/7/7 syllabes respectivement. Cette forme poétique proscrit, en principe, tout recours à une lecture sinisée des termes.

52. Nous avons utilisé les deux éditions de ce texte contenues dans les volumes suivants : Gunshoruijû, Waka-bu, vol. 179, Keizai zasshi-sha, 1900, p. 1116-1117, et Shinpen kokka taikan, vol. 5, Kadokawa-shoten, réed. 1993, p. 876-877.

Sur le Ki no shishô gokusui no en waka, voir, Hashimoto Fumio, Ôchô wakashi no kenkyû, Kasama sho. in, 1972, p. 28-51 ; Yamada Takao, Nihon kagaku no genryû, Nihon sho. in, 1952, p. 5-26 ; Yamaguchi Hiroshi, Ôchô kadan no kenkyûu-Uda, Daigo, Suzaku hen, Ôfûsha, réed.1982, p. 124-128 ; Yoshikawa Eiji, « Kudai-waka no seiritsu to tenkai ni kansuru shiron », Kokubungaku kenkyû, n 68, juin 1979. 
Yamaguchi et Yoshikawa pensent que ce texte est apocryphe et aurait été écrit au milieu ou à la fin de l'époque de Heian. Leurs arguments sont : 1 - les gokusui no en waka n'étaient pas organisés à l'époque ; 2 - il n'est pas d'autre exemple de rencontre poétique à laquelle les huit poètes aient participé ensemble ; 3 - la carrière poétique de Korehira est plus tardive ; 4 - quand il s'agissait de composer des waka en prenant pour sujet des vers chinois, on choisissait des vers célèbres ; 5 - on remarque des similitudes dans l'expression et la conception de certains poèmes de cette manifestation ; 6 - la forme et l'expression des poèmes semblent plus tardive ; 7 - aucun poème de cette manifestation ne figure dans le recueil personnel des huit poètes participants, ni dans les trois premières anthologies compilées sur ordre impérial.

Tous ces arguments peuvent être réfutés et n'ont pas suffi à convaincre l'ensemble des spćcialistes.

53. Dans le Man. yô-shû, anthologie compilée au milieu du VIII ${ }^{\mathrm{e}}$ siècle, sont recueillis trois waka (Livre 19, $\mathrm{n}^{\circ} 4151,4152,4153$ ) composés par Ôtomo no Yakamochi, lors d'un banquet qui se tint le trois du troisième mois dans sa résidence. La référence explicite dans le dernier poème aux coupes que l'on fait flotter en Chine à cette occasion, tout comme la date de la manifestation, attestent qu'il s'agit bien d'un goku sui no en. Nous ne pouvons déterminer en revanche si les participants ont composé tous leurs poèmes en japonais, ou si les poèmes de Yakamochi ne sont que l'appendice d'une série de compositions en chinois.

Un banquet similaire à celui qui nous occupe ici, au cours duquel il s'agissait de composer des waka, se tint le 3 du troisième mois de la première année de Juei (1182) dans la résidence du poète Kamo no Shigeyasu (1119-1191). Deux poèmes composés à cette occasion sont conservés dans le recueil qu'il compila Tsuki môde shûu (n 159 160), col. Shinpen kokka taikan, vol. 2, Éditions Kadokawa, 1974, p. 335.

54. Sur la composition de poèmes japonais sur des sujets/vers extraits de poèmes chinois, pratique appelée kudai waka, on pourra lire notre article : «Les métamorphoses du mot : la citation de vers chinois comme sujet de composition de poèmes japonais, Waka ", publié dans le n 17 de la revue Extrême-Orient, Extrême-Occident, Le travail de la citation en Chine et au Japon, Presses Universitaires de Vincennes, Saint-Denis, 1995 , p. 67-90.

55. L'obscurité est due au fait que, des fleurs couvrant l'eau du rapide, la lune ne peut s'y refléter.

56. Hashimoto, op. cit., p. 40.

57. Nous avons choisi pour ce poème la version contenue dans le Shinpen kokka taikan. Dans le manuscrit du Gunshoruijû l'avant dernier vers est «sayakeku harerul s'éclaircit, limpide ».

58. Shinkokin waka shû, coll. « Shinchô nihon koten shûsei », Éditions Shinchôsha, 1979, vol. 1, p. 68-69.

59. Cité par Monique Mosser et Georges Teyssot, op. cit., p. 13. 
Index des kanji utiles

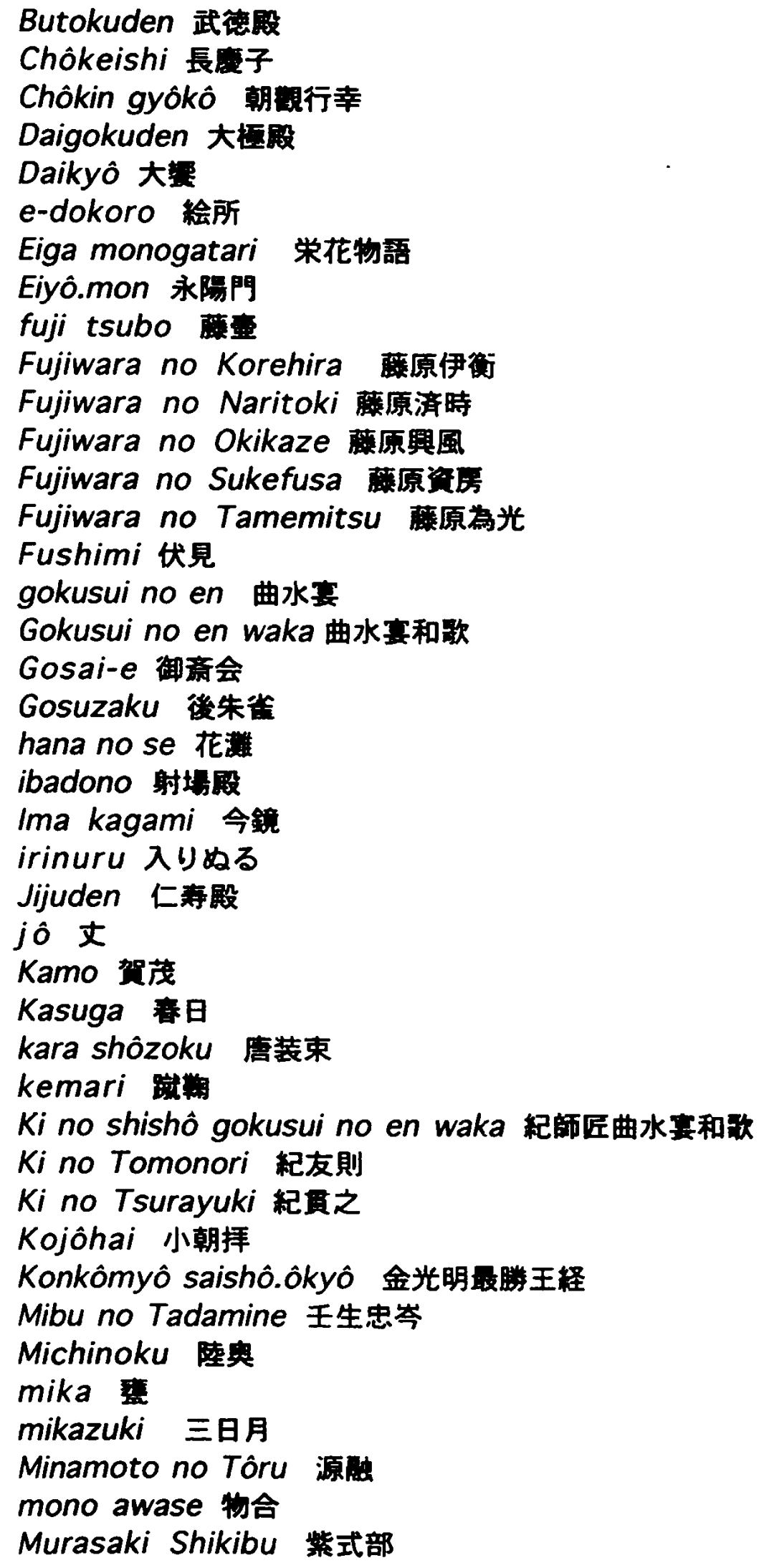




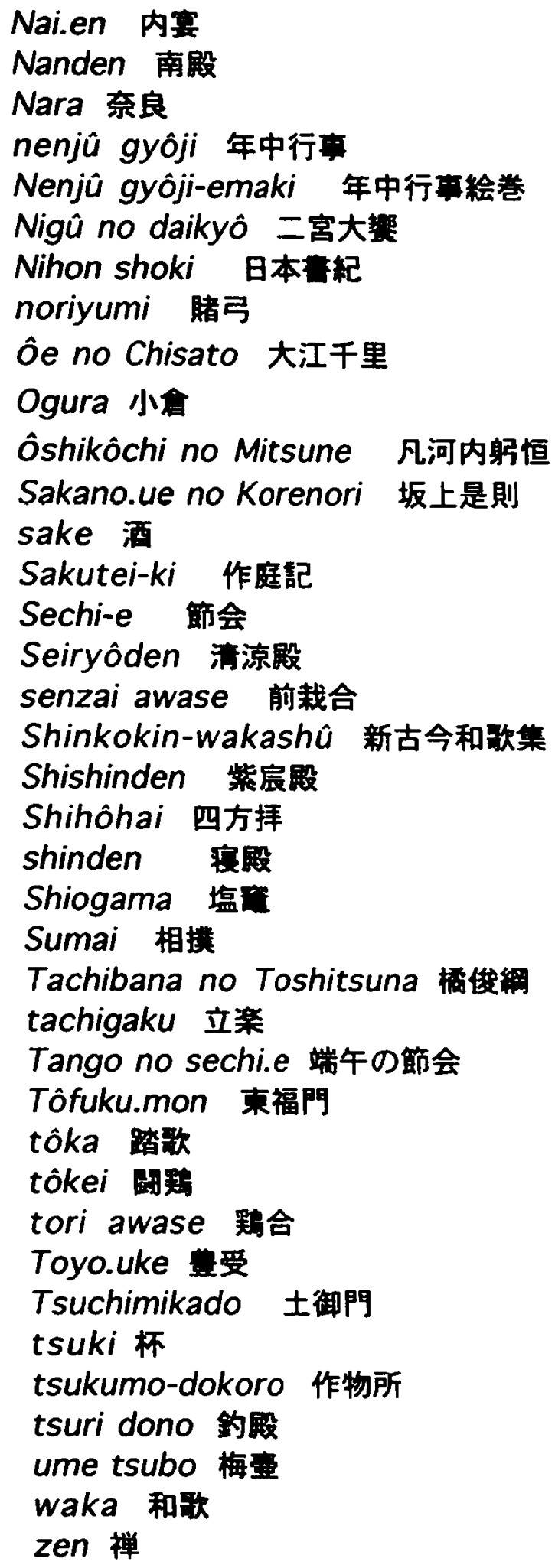

